\title{
Iron Content as an Indicator for Legionella Species in Artificial Water Systems
}

\author{
Rajeshwari Vittal ${ }^{1}$, Juliet Roshini Mohan Raj ${ }^{2}$, Ballamoole Krishna Kumar³ ${ }^{3}$ Indrani Karunasagar ${ }^{4}$ \\ ${ }^{1}$ Senior Lecturer, Nitte University Centre for Science Education and Research, Deralakatte, Mangaluru, \\ India-575018, ${ }^{2}$ Assistant Professor, Division of Infectious Diseases, ${ }^{3}$ Assistant Professor, Division of Infectious \\ Disease, ${ }^{4}$ Director, Nitte University Centre for Science Education and Research, Mangaluru, India-575 018
}

\begin{abstract}
Background: Legionella have emerged as a pathogenic group due to increased use and poor maintenance of artificial water environments. World Health Organization considers Legionella associated diseases to be underestimated, especially in developing countries due to difficulty in detection. The aim of this study was isolation and culture of Legionella spp. from artificial water systems and to test the importance of iron concentration which can be developed as a reliable chemical marker.

Method: Thirty samples each were collected from drinking water coolers, cooling towers and shower heads fitted in different houses of Mangaluru city. Isolation and identification of the bacteria were carried out as per the standard protocols followed by determination of iron content prescribed by IS 3025 (Part 53).

Conclusion: Our study shows that concentrations of iron, is an important factor that increases the likelihood of Legionella spp. Statistically, a concentration of $300 \mathrm{mg} \mathrm{Fe} / \mathrm{L}$ shows a positive correlation with Legionella presence. Therefore, monitoring suspect sites for iron concentration and chelation of available iron, can be developed as respective assays for identifying Legionella spp. Furthermore, we show that PCR based detection of Legionella is a more robust method than the classical 3-plate method, especially for virulent strains.
\end{abstract}

Keywords: Cooling tower, iron content. Legionella, Legionnaires disease, potable water, water distribution systems.

\section{Introduction}

Legionella, a genus of Gram negative, non-spore forming bacilli pervasively occurs in all aquatic habitats and is known to enter into the human-made water systems easily ${ }^{1,2}$. They are known to cause the mild to fatal Legionnaire's diseases (LD) and the milder Pontiac fever both commonly known as Legionellosis.

Among the pathogenic species, L.pneumophila is the most widely studied species; L.longbeachae, L.bozemanii and L.micdadei are other commonly detected agents of Legionellosis ${ }^{3}$.

In developed countries, 2-9 \% of all pneumonia cases are attributed to Legionella infection ${ }^{4-8}$. Lower than expected incidence of L. pneumophila infections from India are believed to be due to under-reporting as most of the infections are diagnosed as atypical pneumonia in community establishments and hospitals ${ }^{7}$.

In the absence of a vaccine, the prime preventive measure forinfections is reduction/elimination of physical sources of infection. However, despite the repeated recommendations of thorough maintenance of the water systems, little scientific evidence exists to show the effect of regular maintenance in reducing Legionella load $^{8}$. Therefore, there is an urgent need to develop novel bio/chemical markers for identification for this group of bacteria or the sites where they can thrive?.

The conventional method for isolation of Legionella spp. includes pre-treatments followed by culturing on buffered charcoal yeast extract agar supplemented 
with cysteine, iron, and antibiotics. In this study, water samples from various municipal sources, we show that the conventional method can give false positive results, therefore, PCR-based detection is a more robust method for detection. Moreover, iron content at the sampled sites and the presence of Legionella spp. show a strong statistical correlation, thereby suggesting that local iron concentration at suspect locations can be used as a chemical marker for probable Legionella growth.

\section{Materials and Method}

Sampling: Ninety samples were collected in total over a period of 2 years, from December 2016 to December 2018 . The study was conducted in Nitte University Center for Science Education and Research, Nitte(Deemed to be University), Deralakatte, Mangaluru. Thirty each samples were collected from local drinking water coolers, cooling towers and from shower heads fitted in different houses around Mangaluru city. samples were collected by strictly following the Indian Standard: Method of sampling and test for water and wastewater guidelines. All samples were processed within 4 hours of collection.

Isolation and culturing of samples: Isolation of Legionella from collected samples was performed as per US Centre for Disease Control and Prevention guidelines 2005. Briefly, each water samples were filter concentrated in a biological safety cabinet by pouring the samples onto a sterile membrane filtration funnel containing $0.2 \mu \mathrm{m}, 45 \mu \mathrm{mm}$ diameter polycarbonate membrane filter. After filtration, the filter was removed aseptically from the holder with sterile filter forceps and placed into a centrifuge tube containing $5 \mathrm{ml}$ sterile water. The centrifuge tube was then vortexed for 1 minute to recover bacteria and organic material from the filter. Buffered charcoal yeast extract (BCYE) supplemented with glycine, vancomycin, cycloheximide and polymyxin B (GVCP) antibiotics was used for isolation of Legionella. 2 BCYE plates and GVCP plates were inoculated with $100 \mu 1$ suspension each and spread with a sterile spreader. The plates were incubated at 35 ${ }^{\circ} \mathrm{C}$ in a candle jar for 72 hours.

Legionella pneumophila ATCC 33152 procured from LGC Promochem, Bangalore was used as a positive control for all the experiments. The lyophilized culture was resuscitated as per manufacturer's instructions.

PCR for detection of Legionella species: Template DNA was prepared by centrifuging the growth suspension at $12000 \times \mathrm{g}$, for 5 minutes . The supernatant was discarded and the pellet uniformly resuspended in $100 \mu 1$ sterile $100 \mathrm{mM}$ Tris-EDTA by gentle vortexing. The resuspension was placed in a dry bath at $98^{\circ} \mathrm{C}$ for 10 minutes and then flash cooled on ice. the cooled resuspension was used as the DNA template without further purification. $10 \mathrm{pM}$ standard 16S JFP species specific primers were used for the detection of Legionella pneumophila and Legionella spp. Further, extracted DNA was used in a nested PCR for virulence detection by dot (defective organelle trafficking) amplification.

Determination of iron content: The iron content of each sample was measured by 1,10 phenanthroline method as prescribed by IS 3025 (Part 53).

\section{Results and Discussion}

Legionella infections are primarily spread by manmade water systems and devices such as showers, whirlpool spas, and cooling towers which release contaminated aerosols. Inhalation of these aerosols especially by those with compromised immune systems leads to the infection ${ }^{10}$. WHO predicts that incidences of Legionella related disease outbreaks are under-reported in developing countries due to lack of clinical awareness. Reports of Legionella pneumophila incidence from India are limited in number and are mostly from clinical samples. Our study primarily aimed at determining the prevalence of Legionella pneumophila and its related species in the geographical location of Mangaluru, India and to determine chemical indicators. Isolation of Legionella species by culture technique and enrichment followed by molecular detection was performed for each sample . According to CDC protocol, Legionella spp. is positive on both BCYE and GVPC plates. Growth on GVPC plates is a unique characteristic of Legionella spp. and is used as confirmatory test, as other species are not known to grow on it. Cysteine deficient GVPC plates are used as a control, since cysteine supplement is essential for Legionella growth. Based on morphological features, both the agar plates in all the samples, predominantly showed round pearl-white colonies (Fig 1). Thus, suggesting that all the samples were positive for Legionella (Table 1). However, some control samples without cysteine supplement also showed Legionella like colonies on the agar plates. Repeated experiments showed growth of Legionella like colonies in a fraction of control samples, indicating experimental design problem. In our experiments, the 3-plates method for definitive identification of Legionella based on growth 
characteristics, provided a fraction of false positive results. This demands for a more robust method for detection of Legionella spp. Thus, standard PCR with species specific primers and nested PCRs were set with dot gene specific primers to check the virulence of the samples (Table S1).

Agarose gel-electrophoresis of the PCR products showed that from drinking water coolers, of the 30 samples, only 1 sample was tested positive and, 9 samples showed positive from cooling towers. Out of 29 tested positive in culture method from showerheads only 2 samples of 30 were positive (Fig 2). All the samples showing positive PCR for JFP-16S also showed positive for virulence gene (Figure 2 and Table 2). Thus, PCR method showed a higher specificity than culturing method in detection of Legionella spp.

Cooling towers are a part of the air-conditioning systems present in industries and hospitals, which use water to efficiently cool air via heat transfer ${ }^{4,10,11}$. The proportion of Legionella culture-positive cooling towers that have been reported from surveys conducted in Asia, Australia, Europe, and the United States is approximately $40 \%{ }^{8,12}$. In this study, about a one third of the samples (30\%) were positive for Legionella spp. by highly sensitive PCR detection and all the samples (30\%) harboured the virulence genes used in the study.

Legionella spp. have been frequently isolated from hospital shower heads but the question of whether aerosols of shower water or other exposures to potable water containing $L$. pneumophila may cause Legionnaires disease is yet unresolved. There have been limited reports on the presence and implications of Legionella in domestic shower heads. Shower heads from residences across Mangaluru reported 9 of 30 samples positive for Legionella. Drinking water is another source that is not generally checked for Legionella since the pathogen is generally contracted by aerosol spray. On an experimental basis, we looked into the presence of Legionella in drinking water from coolers and found 1 of 30 samples positive. The low temperatures of water in these coolers could probably be controlling the growth of this organism. However, the presence of Legionella in water that is considered potable is still a public health concern.

The biggest hurdle in testing for this organism is its fastidious nature. The conventional culturing technique is gold standard but takes as long as 14 days to confirm a result. Rapid detection method are the need of the hour for disease prevention and management of outbreaks if any ${ }^{13}$. Modern techniques like polymerase chain reaction, immunofluorescence and flow cytometry have been reported for rapid detection, but the cost of routine testing and availability of these facilities in testing laboratories across developing countries is very limited. Chemical parameter testing like estimation of iron is a relatively simple method that can be carried out without any requirement of sophisticated equipment and the process takes less than 4 hours to report the result.

To characterize Legionella harbouring surfaces, we looked for essential nutrients that Legionella needs for survival and flourishing. From the list of nutritional elements in Legionella culture medium, iron is an essential nutrient that can be easily detected and quantified by sensitive assays. Further, since iron is freely available in the environment in many different forms it can be expected to be a promising candidate as a chemical marker for the development of rapid detection of Legionella. Iron is an essential nutrient for L. pneumophila because its growth depends on the presence of iron in its culture medium and also plays a key role in its pathogenesis ${ }^{14}$. Recently there have been reports to implicate that the presence of cast iron rusting plays an important role in the development of Legionella in water distribution systems.

Interestingly, majority of the sites tested in our study had iron concentration higher than $300 \mathrm{mg} / \mathrm{l}$. Routine BCYE and GVPC media used to culture Legionella is supplemented with $250 \mathrm{mg} / \mathrm{l}$ of iron for optimal growth. Among the different sites, cooling tower samples showed the highest proportion, at $80 \%$ of samples with iron concentration $>300 \mathrm{mg} / \mathrm{ml}$. Drinking water coolers and showerheads showed high iron concentrations in 53 and $43 \%$ samples respectively (Table 3). This proportionality of high iron concentration corelates with PCR positive Legionella samples, where cooling towers showed highest number of positive samples at $30 \%$ while drinking water coolers and showerheads showed 2 and $6 \%$ PCR positive samples, respectively (Table 4). It is noteworthy that all the PCR positive Legionella samples had iron concentration higher than $75 \mathrm{mg} / \mathrm{l}$ showing that low environmental iron concentrations are not conducive for growth. As hypothesized iron could be used as an indicator for Legionella in routine testing of water. Our study indicates that while concentrations up to $75 \mathrm{mg} / \mathrm{l}$ do not show a correlation with the presence of Legionella but $300 \mathrm{mg} / \mathrm{l}$ iron concentration is a strong 
indicator of Legionella and thus can be used for routine monitoring.

Our study also shows that environmental monitoring should be an important and regular practice for preventive measures, which could begin with sites that are likely to have high local iron concentration. The
WHO recommends that health care facilities should have a general water safety plan as a part of infection control and these plans should address microbial growth in addition to control of external contamination by Legionella and should include ancillary equipment like shower heads and medical devices.

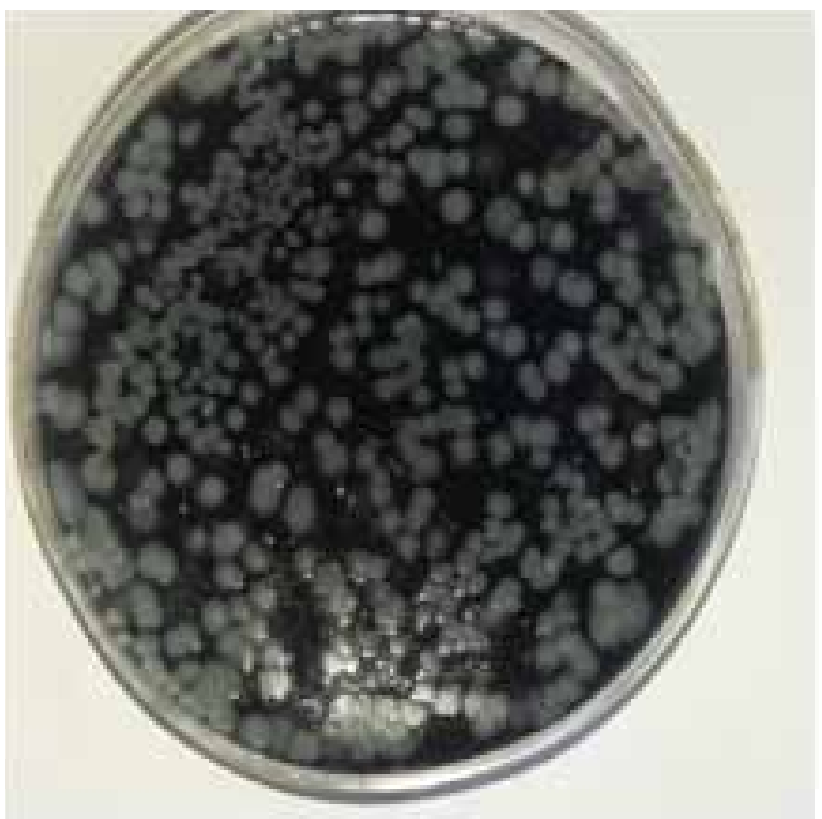

A

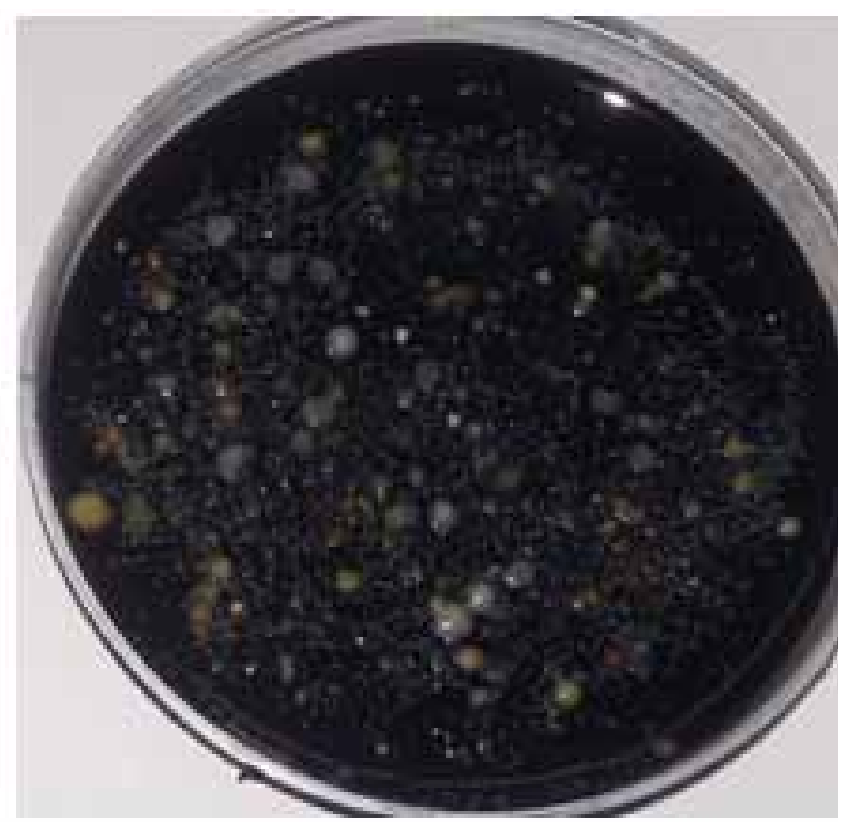

B

Figure 1: Representative Legionella spp. cultures, showing typical round pearl-white colonies on (a) GVPC and (b) BCYE agar plates.

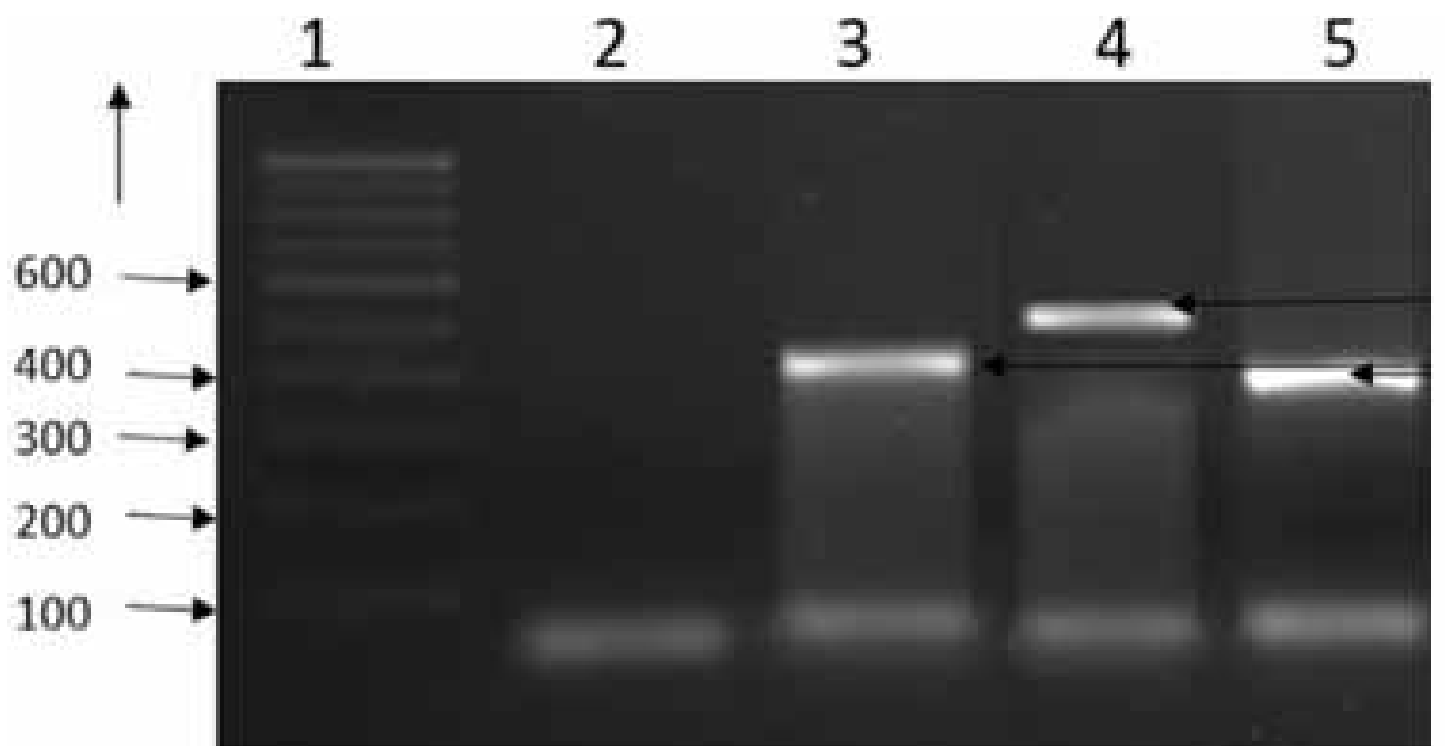

Figure 2. Representative agarose gel electrophoresis of one of the cooling tower samples. Lane 1: DNA ladder, lane 2: negative control, lane 3: JFP gene with expected size of $386 \mathrm{bp}$, lane 4: dot A step 1 product with expected size of $440 \mathrm{bp}$, lane 5: dot $\mathrm{F}$ step 2 product with expected size of $387 \mathrm{bp}$. 
Table 1: Summary of Legionella positive cultures based on 3-plate method.

\begin{tabular}{|l|c|c|c|c|}
\hline Sample Source & No. of samples & $\begin{array}{c}\text { No. of positive } \\
\text { BYCE plates }\end{array}$ & $\begin{array}{c}\text { No. of positives } \\
\text { GVPC plates }\end{array}$ & $\begin{array}{c}\text { No. of positive } \\
\text { GVP(w/cysteine)-plates }\end{array}$ \\
\hline Drinking water coolers & 30 & 30 & 30 & 10 \\
\hline Cooling towers & 30 & 30 & 29 & 15 \\
\hline Showerheads & 30 & 30 & 30 & 30 \\
\hline
\end{tabular}

Table 2: Summary of Legionella positive samples from different sources.

\begin{tabular}{|l|c|c|}
\hline Sample source & Growth on GVCP & PCR \\
\hline Drinking water coolers & 30 & 1 \\
\hline Cooling towers & 29 & 9 \\
\hline Showerheads & 30 & 2 \\
\hline
\end{tabular}

Table 3: Iron concentration range from samples across different sites.

\begin{tabular}{|c|c|c|c|}
\hline Source & $<75 \mathrm{mg} / \mathrm{l}$ & $75-300 \mathrm{mg} / \mathrm{l}$ & $>300 \mathrm{mg} / \mathrm{l}$ \\
\hline Drinking water coolers & 5 & 9 & 16 \\
\hline Cooling towers & 1 & 5 & 24 \\
\hline Showerheads & 5 & 12 & 13 \\
\hline
\end{tabular}

Table 4: Iron concentration of PCR positive Legionella samples.

\begin{tabular}{|c|c|c|}
\hline Sample ID & Sample source & Iron concentration (mg/l) \\
\hline K5 & Drinking water & 80 \\
\hline CT1 & Cooler water & 731 \\
\hline $\mathrm{CT} 2$ & Cooler water & 731 \\
\hline CT7 & Cooler water & 3300 \\
\hline CT15 & Cooler water & 1299 \\
\hline CT16 & Cooler water & 567 \\
\hline CT17 & Cooler water & 75 \\
\hline CT27 & Cooler water & 92 \\
\hline CT28 & Cooler water & 92 \\
\hline СТ29 & Cooler water & 153 \\
\hline G1 & Shower head & 136 \\
\hline G6 & Shower head & 200 \\
\hline
\end{tabular}

Table S1: List of primers.

\begin{tabular}{|l|l|c|}
\hline Primer & Sequence 5'-3' & Amplicon (size bp) \\
\cline { 1 - 2 } JFP & AGGGTTGATAGGTTAAGAGC & 386 \\
\cline { 1 - 2 } JRP & CCAACAGCTAGTTGACATCG & \multirow{2}{*}{440} \\
\hline dotF & ATTGTCTCGCGCGATTGC & 387 \\
\hline dotRM & CTTCCATTGAGTTTCACCAAATCA & \multirow{2}{*}{3} \\
\hline dotFK & GGTGATGGTTAATAATGATCCGGC & \\
\hline
\end{tabular}




\section{Conclusion}

Legionella species were found to be prevalent in artificial water systems. The misconception that Legionella related diseases occur in developed countries alone should be overcome by public awareness and routine environmental monitoring. Our study indicates that $300 \mathrm{mg} \mathrm{Fe} / \mathrm{L}$ is a reliable indicator of Legionella in case of cooling towers and thus can be used as first line of testing for potential sites for Legionella growth.

\section{Conflict of Interest: Nil}

Sources of Funding: Nitte (Deemed to be University) through the faculty research grant NURG/ STF/07/7-2015.

Ethical Clearance: Obtained from K.S HEGDE MEDICAL ACADEMY Institutional Ethics Committee, Reference No: INST.EC/EC/021/2015-2016.

\section{References}

1. Steinert M, Hentschel U, Hacker J. Legionella pneumophila: an aquatic microbe goes astray. FEMS microbiology reviews. 2002 Jun 1;26(2):149-62.

2. Newton HJ, Ang DK, Van Driel IR, Hartland EL. Molecular pathogenesis of infections caused by Legionella pneumophila. Clinical microbiology reviews. 2010 Apr 1;23(2):274-98.

3. Long SS, Pickering LK, Prober CG. Principles and practice of pediatric infectious diseases, Elsevier Health Sciences. Philadelphia, PA. [Google Scholar]. 2012.

4. Parr A, Whitney EA, Berkelman RL. Legionellosis on the rise: a review of guidelines for prevention in the United States. Journal of Public Health Management and Practice. 2015 Sep;21(5):E17.

5. Chaudhry R, Dhawan B, Dey AB. The incidence of Legionella pneumophila: a prospective study in a tertiary care hospital in India. Tropical doctor. 2000 Oct;30(4):197-200.

6. Chaudhry R, Sreenath K, Arvind V, Vinayaraj EV, Tanu S. Legionella pneumophila serogroup
1 in the water facilities of a tertiary healthcare center, India. Emerging infectious diseases. 2017 Nov;23(11):1924.

7. Anbumani S, Gururajkumar A, Chaudhury A. Isolation of Legionella pneumophila from clinical $\&$ environmental sources in a tertiary care hospital. Indian Journal of Medical Research. 2010 Jun 1;131(6):761.

8. Llewellyn AC, Lucas CE, Roberts SE, Brown EW, Nayak BS, Raphael BH, Winchell JM. Distribution of Legionella and bacterial community composition among regionally diverse US cooling towers. PloS one. 2017 Dec 20;12(12):e0189937.

9. Atlas RM. Legionella: from environmental habitats to disease pathology, detection and control. Environmental Microbiology. 1999 Aug;1(4):28393.

10. Leoni E, De Luca G, Legnani PP, Sacchetti R, Stampi S, Zanetti F. Legionella waterline colonization: detection of Legionella species in domestic, hotel and hospital hot water systems. Journal of Applied Microbiology. 2005 Feb;98(2):373-9.

11. $\mathrm{Xu} \mathrm{L}$, Luo ZQ. Cell biology of infection by Legionella pneumophila. Microbes and infection. 2013 Feb 1;15(2):157-67.

12. Türetgen I, Sungur EI, Cotuk A. Enumeration of Legionella pneumophila in cooling tower water systems. Environmental monitoring and assessment. 2005 Jan 1;100(1-3):53.

13. Benowitz I, Fitzhenry R, Boyd C, Dickinson M, Levy M, Lin Y, Nazarian E, Ostrowsky B, Passaretti T, Rakeman J, Saylors A. Rapid identification of a cooling tower-associated legionnaires' disease outbreak supported by polymerase chain reaction testing of environmental samples, New York City, 2014-2015. Journal of environmental health. 2018 Apr;80(8):8.

14. Reeves MW, Pine L, Hutner SH, George JR, Harrell WK. Metal requirements of Legionella pneumophila. Journal of clinical microbiology. 1981 Apr 1;13(4):688-95. 